\title{
ELECTRIC PROPULSION SYSTEMS \\ FOR SMALL SATELLITES: THE LOW EARTH ORBIT MISSION PERSEUS
}

\author{
D. Bock $^{1}$, G. Herdrich ${ }^{1}$, M. Lau ${ }^{1}$, M. Lengowski ${ }^{1}$, \\ T. Schönherr ${ }^{2}$, F. Steinmetz ${ }^{1}$, B. Wollenhaupt ${ }^{1}$, O. Zeile ${ }^{1}$, \\ and H.-P. Röser ${ }^{1}$
}

\author{
${ }^{1}$ Institut für Raumfahrtsysteme (IRS) \\ Universität Stuttgart \\ Pfaffenwaldring 31, Stuttgart 70569, Germany \\ ${ }^{2}$ Department of Advanced Energy \\ University of Tokyo
}

Kashiwa-no-ha 5-1-5, Kashiwa 277-8561, Japan

The Institute of Space Systems, Universität Stuttgart, launched a "Small Satellite Program" in 2002. The first two of the four planed small satellites, Flying Laptop and PERSEUS, are both Low Earth Orbit (LEO) missions. The third mission Cermit is a reentry satellite and the last of the small satellites - Lunar Mission BW1 — is a mission to the Moon. For this purpose, different propulsion systems are mandatory. The propulsion system for Lunar Mission BW1 will consist of two different types of thruster systems: a cluster of pulsed magnetoplasmadynamic (MPD) thrusters (SIMP-LEX) using solid polytetrafluoroethylene (PTFE) as propellant and a thermal arcjet thruster (TALOS) using gaseous ammonia as propellant. Both thruster systems are currently under development at IRS. They are planned to be tested on board the small satellite mission PERSEUS, one of the precursor missions of Lunar Mission BW1. The thruster systems have been investigated intensely in the past and, furthermore, optimization of the thrusters with respect to the mission requirements of Lunar Mission BW1 has been started. The test procedures for the technology demonstration on the PERSEUS satellite are under development at present.

\section{INTRODUCTION}

Within the "Small Satellite Program" at IRS, Universität Stuttgart, consisting of four small-satellite missions, two low-cost electric thruster systems are devel-

This is an Open Access article distributed under the terms of the Creative Commons Attribution-Noncommercial License 3.0, which permits unrestricted use, distribution, and reproduction in any noncommercial medium, provided the original work is properly cited. 
oped - the pulsed plasma thruster (PPT) SIMP-LEX and the thermal arcjet thruster TALOS [1, 2]. These systems are planned to be used as primary propulsion for the IRS small-satellite mission Lunar Mission BW1, which will orbit the Moon in a polar orbit with an altitude of about $100 \mathrm{~km}[3]$.

The flight to the Moon is expected to take about two years and is divided into four different mission phases.

Phase I begins after insertion into geostationary transfer orbit (GTO) and ends after the spacecraft's perigee is raised above the outer van-Allen-belt. Flight phase II lasts until the Moon's sphere of influence is reached. Phase III ends with the insertion into a stable elliptical lunar capture orbit and during the final mission phase, the satellite is injected into its final orbit around the Moon.

The thrust demands during mission phases I and III are higher than during the other mission phases. Therefore, the arcjet thruster as the highest thrust unit will be used during these phases. During the other mission phases solely, the pulsed MPD cluster will be used for propulsion. The in-orbit validation of those thruster systems is accomplished with the second of the four small satellites, PERSEUS.

The PERSEUS satellite will have a mass of about $150 \mathrm{~kg}$ and provide about 1-kilowatt electrical power. The dimensions of the cube-shaped satellite will be $50 \times 70 \times 85 \mathrm{~cm}$. It is scheduled to be launched into a polar, sun-synchronous LEO below $1000 \mathrm{~km}$. Its mission is planned to be divided into two stages: at the first, the two propulsion systems will be tested and validated, and at the second, ultraviolet (UV) astronomy observations with a telescope and a spectrometer with a charge-coupled device (CCD) sensor will be made. The sensor will be sensitive in the spectral band of 120-180 nm. In this part of the spectral band, many astronomical phenomena like supernovae can be observed by PERSEUS in a way that is not possible from the Earth. The UV-astronomy is planned to be performed in cooperation with the Institut für Astronomie und Astrophysik Tübingen.

This paper focuses on the current status in the development of electric thrusters SIMP-LEX and TALOS at IRS. Furthermore, it gives a short overview of the PERSEUS satellite as well as the test and validation procedures of the thruster systems as planned during the PERSEUS mission.

\section{PULSED PLASMA THRUSTER}

A PPT is an MPD electric propulsion system. This means, it uses the electric power provided by a spacecraft to accelerate propellant by means of the electromagnetic Lorentz force. As only charged particles can be accelerated, the ablated propellant is transformed into ionized plasma. To maximize the power supplied 


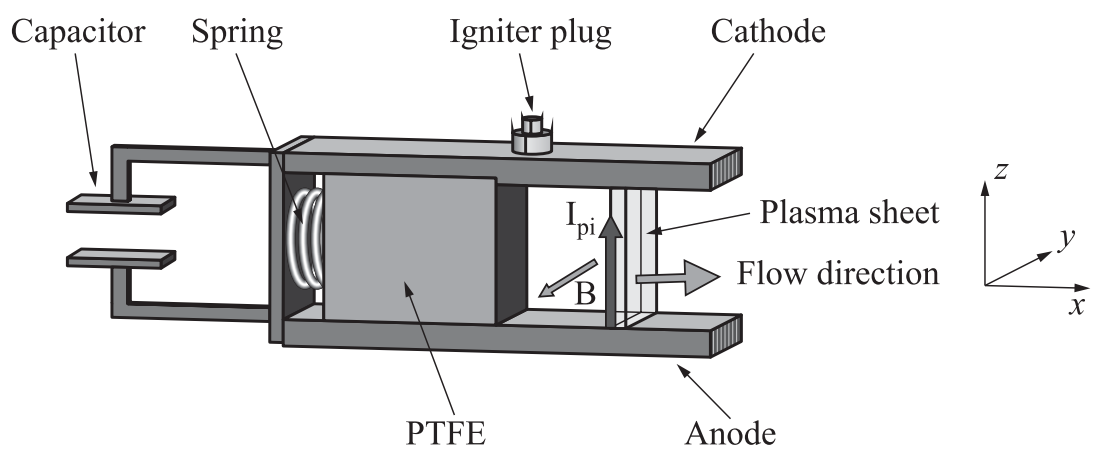

Figure 1 Operation principle of a PPT

to the plasma while keeping thermal loads at a tolerable level, the plasma is created by a high-current discharge pulse. As a result, the thruster assembly represents an electric circuit. The parts and operation principle are presented in Fig. 1.

The PPT consists of four main parts: capacitor, electrodes, propellant feed system, and igniter plug. The capacitor is needed to store electric energy for a discharge pulse. The two electrodes, anode and cathode, are connected to the capacitor terminals to carry their electric potential. A block of solid PTFE propellant is placed between the electrodes and fed by a mechanic feed system, for example, by a spring as shown in Fig. 1. Prior to a discharge pulse, a voltage is applied to the capacitor. A pulse is started by firing the igniter plug. The discharge of the capacitor ablates a small amount of PTFE at the propellant surface that forms a plasma sheet. The electric circuit is now closed by the capacitor, electrodes, and plasma sheet. The current through the plasma sheet creates a self-induced magnetic field. Interaction of the ionized particles of the current with this field causes the electromagnetic Lorentz force and expels the propellant particles to provide thrust.

In the past decades, numerous PPTs have successfully been operated for the purpose of technology demonstration, north-south station keeping, highprecision target pointing as well as drag compensation $[4,5]$. Their robust and simple design is a clear advantage in terms of safety and reliability while the pulsed operation allows for very flexible power management.

At IRS, the PPT SIMP-LEX is developed as the primary propulsion system of the Lunar Mission BW1 and for technology demonstration on the PERSEUS satellite. The thruster has been theoretically and experimentally investigated at IRS as part of the project funded by the Deutsches Zentrum für Luft und Raumfahrt (DLR) in the past. Recently, extensive development efforts by Nawaz et al. in the field of thruster optimization in combination with close cooperation with 


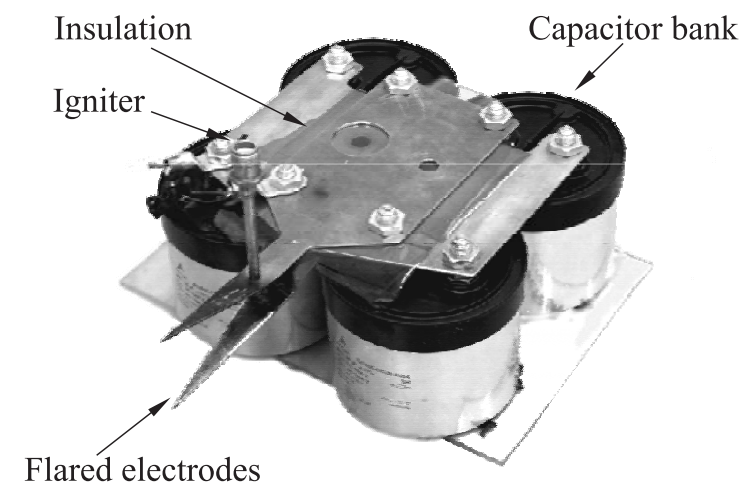

Figure 2 ADD SIMP-LEX thruster

the Russian partner — Research Institute of Applied Mechanics and Electrodynamics of Moscow Aviation Institute (RIAME MAI) have led to the introduction of the ADD SIMP-LEX thruster [6, 7].

The design was optimized to improve thrust efficiency and to further allow for a closer study of the dependence of the SIMP-LEX thruster on its electrical parameters. A picture of the thruster is given in Fig. 2.

Measurements of the ADD SIMP-LEX performance resulted in a specific impulse of $2500 \mathrm{~s}$ and a mass bit of $53 \mu \mathrm{g}$ at a pulse frequency of $0.5 \mathrm{~Hz}$. However, the pulse frequency on PERSEUS will be closer to $1 \mathrm{~Hz}$.

Currently, development efforts are ongoing at IRS to characterize performance and electrical parts as well as life testing of the thruster for optimization and eventually integration of the SIMP-LEX flight model. In the next step, the propellant feeder, electronic control, and telemetry for on-orbit testing will be integrated for subsequent acceptance testing of the PERSEUS protoflight unit. The second testbed for the development of the thruster has been established at the University of Tokyo in Japan to provide data for the simulation of the discharge pulse and for plasma diagnostics.

\section{THERMAL ARCJET THRUSTER}

A thermal arcjet thruster is the electric propulsion system where the propellant is heated up by an electric arc and accelerates in a Laval nozzle. The operation principle and main parts of the TALOS arcjet are shown in Fig. 3. The thruster consists of an annular anode, which is the thruster nozzle, and a central cathode as well as the injector, which is furthermore the electrical insulation between the electrodes. For ignition, the propellant flow is opened and a high 


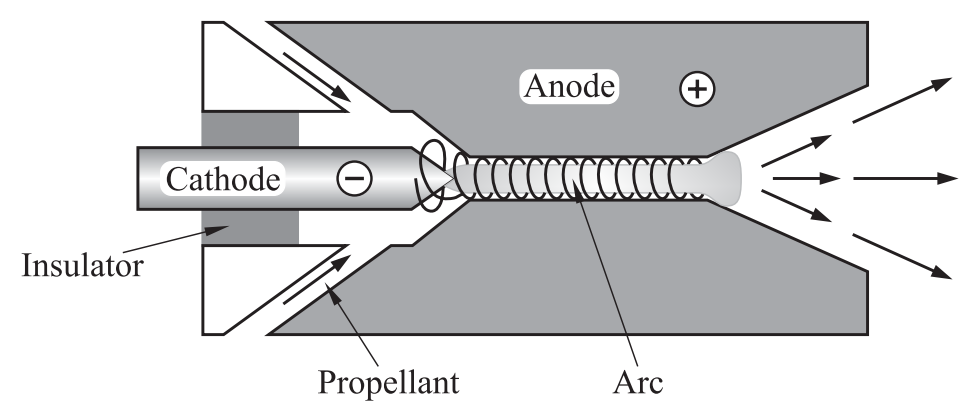

Figure 3 Operation principle of thermal arcjet thruster

discharge voltage on the level of $2000 \mathrm{~V}$ is applied to the cathode and anode. Ignition is accomplished via a Paschen-discharge. Once ignited, the arc between cathode and anode heats up the propellant causing its dissociation and ionization.

The propellant is then expanded in the nozzle converting the thermal energy supplied to the propellant by heating into the kinetic energy of the directed flow. As a result, the thrust is generated.

As the exhaust velocity of thermal arcjet is inversely proportional to the molecular weight of the propellant, light propellants are preferred to achieve a higher exhaust velocity and, hence, thrust.

These include hydrogen and propellants with a high component of hydrogen like hydrazine and ammonia. For reasons of storability, hydrogen is only used for ground testing, whereas hydrazine is used in flight applications like north-south station keeping for geostationary satellites [8]. The first on-orbit experiments with a 26-kilowatt thermal arcjet thruster using ammonia as propellant have been successfully accomplished by Bromaghim et al. [9]. For the thermal arcjet thruster of the Lunar Mission BW1, ammonia is planned to be used as propellant as well.

Figure $4 a$ shows a sectional drawing of the thruster laboratory model and Fig. $4 b$ demonstrates the thruster during operation with ammonia. During the development process of the thermal arcjet thruster, the cathode gap (the distance between cathode and anode) can be adjusted. Also, the nozzle geometry can be changed by keeping the outer nozzle geometry constant and changing the inner geometry (like the nozzle throat diameter, nozzle throat length, and expansion angle).

The cathode is made of $2 \%$ thoriated tungsten and is $3 \mathrm{~mm}$ in diameter. The cathode tip is a cone with $22.5^{\circ}$ half-cone angle. During the optimization process of the thruster aimed at meeting the requirements of the Lunar Mission BW1, different nozzle geometries as well as different nozzle materials were investigated. 


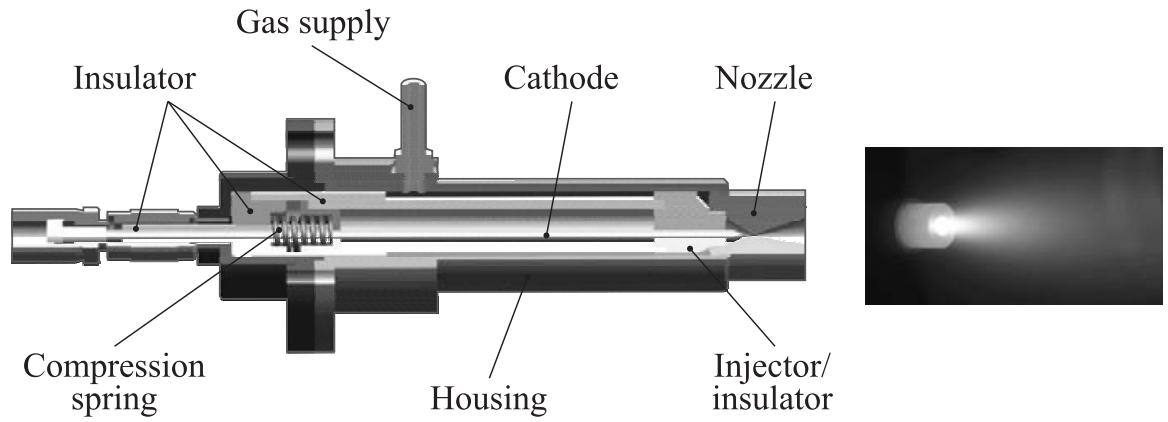

(a)

(b)

Figure 4 Sectional drawing of thruster $(a)$ and thruster during operation $(b)$

These materials are tungsten alloys with thorium oxide, lanthanum oxide, and rhenium. The geometry of the nozzle was varied with the nozzle throat between 0.4 and $0.6 \mathrm{~mm}$, nozzle throat length between 0.4 and $1 \mathrm{~mm}$, and the expansion half-angle between $19^{\circ}$ and $20^{\circ}$, the converging half-cone angle of all investigated nozzle geometries was $35.2^{\circ}$. The thruster was radiation cooled.

In general, the performance characteristics of arcjet thrusters depend on the total energy input, mass flow rate, and the propellant used. For a nozzle geometry with a throat diameter of $0.6 \mathrm{~mm}$, throat length of $0.7 \mathrm{~mm}$, and expansion angle of $40^{\circ}$, the mean performance for $30 \mathrm{~h}$ of operation with ammonia was as follows: $800 \mathrm{~W}$ electrical input power and $26 \mathrm{mg} / \mathrm{s}$ mean mass flow rate. The thrust level achieved was $110 \mathrm{mN}$ for this operating conditions at stationary operation. The thruster was operated as planned for the Lunar Mission BW1: one-hour stationary operation mode followed by one-hour battery charge mode with no thruster operation. The nozzle material used for this investigation was tungsten-thorium oxide.

The propellant feed system of the thermal arcjet thruster supplied gaseous ammonia at constant mass flow rate and pressure to the thruster. The mass flow was controlled by the pressure drop over the flow aperture. Therefore, the pressure in front of the flow aperture was set by the pressure regulator [10]. As ammonia was stored inside the propellant tank in liquid phase, a heat exchanger was used to heat up and vaporize it prior to injection into the thruster. Main parts of the propellant feed system are the tank, a check valve to open and close the propellant flow, a vaporizer, a pressure regulator, and flow aperture. Impurities are filtered out by two T-type filters with a pore size of 15 and $7 \mu \mathrm{m}$, respectively. Currently, investigations under vacuum conditions for characterization of a cocurrent microchannel heat exchanger are conducted. The microchannel heat exchanger is developed by the Institute of Micro Process Engineering of the Forschungszentrum Karlsruhe in der Helmholzgesellschaft [11]. 


\section{SMALL-SATELLITE MISSION PERSEUS}

The PERSEUS satellite is one of the precursor missions for Lunar Mission BW1. It will be a cube-shaped satellite with a mass of about $150 \mathrm{~kg}$ and about 1-kilowatt electric peak power. The dimensions of the satellite will be $50 \times 70$ $\times 85 \mathrm{~cm}$. It will be launched into a polar, sun-synchronous LEO below $1000 \mathrm{~km}$. As was mentioned above, its mission will be divided into two stages including propulsion tests and UV-astronomy.

The preliminary design of the PERSEUS satellite is shown in Fig. 5. Both thrusters are located at the bottom of the satellite, the thermal arcjet thruster in the center and the pulsed plasma thruster at the left.

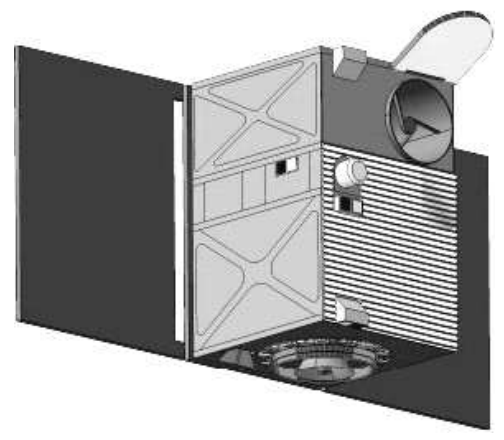

Figure 5 PERSEUS satellite

Currently, the early developments of the attitude control system (ACS) algorithms for PERSEUS are underway. Due to the two very different mission objectives, it is a demanding task to design an ACS that satisfies all mission requirements. For the propulsion tests, one could expect high disturbance torques from the thrusters. However, this phase can deal with a very moderate pointing accuracy. The exact opposite is the case for the UV-astronomy phase. The ACS has to deal with very low disturbance torques that mainly originate from aerodynamic, gravitational, and magnetic disturbances. The pointing requirement of $7 \operatorname{arcsec}$ is quite demanding. Another requirement is that the ACS of PERSEUS should be built on the heritage of the first Stuttgart smallsatellite mission concerning the hardware and parts of the ACS algorithms. The early work and simulations show quite promising results. With the addition of a fine guidance sensor, PERSEUS should be able to fulfill the pointing requirements.

The Lunar Mission BW1 will feature a cluster of minimum four SIMP-LEX thruster units to provide the required thrust and total impulse needed to reach and orbit the Moon. However, the PERSEUS satellite is capable of hosting 
only one PPT thruster together with the propellant feeding system needed to store solid PTFE propellant inside the Lunar Mission BW1 satellite. Hence, all verification tests on PERSEUS will be performed using a single SIMP-LEX unit. As for all PPTs with solid PTFE propellant, the pulse frequency of the thruster is limited due to thermal self-depolymerization effects of PTFE, also limiting the fuel consumption rate. This constraint inevitably results in a long mission duration for the Lunar Mission BW1 which extends the overall operation time of the PERSEUS thruster testbed. Nevertheless, the testing of an entire SIMPLEX lifecycle of two to three years of continuous operation remains a challenging requirement and is not going to be carried out with the PERSEUS mission but inside the ground test facilities at IRS. The on-orbit test program of the SIMPLEX flight demonstration mission is planned to be carried out in three phases:

(i) a test phase for the study of the thruster operation and health;

(ii) a verification phase for assessing spacecraft compatibility; and

(iii) a phase for spacecraft maneuvering.

The flight system of the thermal arcjet TALOS thruster on PERSEUS will consist of the thruster system as developed for Lunar Mission BW1 but with less propellant and thus a smaller propellant tank. For the verification of the thruster operating cycles of $1 \mathrm{~h}$ of arcjet firing mode and $1 \mathrm{~h}$ of battery charging mode as planned for the Lunar Mission BW1 are planned to be carried out. Again, full lifetime tests are not accomplished on the PERSEUS mission but inside the test facilities at IRS. During the PERSEUS mission, a total test time of $30 \mathrm{~h}$ of arcjet thruster operation is scheduled to be carried out.

Depending on the orbit and the configuration of the satellite, the solar panels cannot be oriented directly towards the Sun. This means that the limiting factor for the continuous arcjet firing on PERSEUS in orbit will be the available energy capacity of the satellite batteries. The batteries of PERSEUS will be sized in a way that they allow the continuous arcjet operation for $1 \mathrm{~h}$.

\section{CONCLUDING REMARKS AND FUTURE WORK}

The small-satellite mission PERSEUS will be a technology demonstration testbed for the two electric propulsion systems, which are going to be used for Lunar Mission BW1, the pulsed MPD SIMP-LEX thruster, and the thermal arcjet TALOS thruster. At present, the development of these propulsion systems is accomplished at IRS. The optimization process of both thruster systems has been started and for the thermal arcjet thruster, a 30-hour subscale lifecycle test has been conducted on ground. 
A detailed test program for the in-orbit verification as well as a diagnostic system to gather the necessary information on thruster operation on-board the PERSEUS satellite is under development. During the verification procedure, orbit maneuvers as foreseen for the Lunar Mission BW1 are planned to be executed with both thruster systems separately. Prior to these maneuvers, the checkout of the thruster systems and sensor systems will be accomplished to inspect for malfunctions. After the thruster tests are completed, the PERSEUS satellite will accomplish UV-astronomy observations in the spectral range of 120-180 nm in cooperation with Institut für Astronomie und Astrophysik Tübingen.

\section{ACKNOWLEDGMENTS}

The funding of the PhD of Birk Wollenhaupt by Carl-Zeiss-Stiftung, Oliver Zeile and Fabian Steinmetz by Landesgraduiertenförderung Baden-Württemberg, Michael Lengowski by Konrad-Adenauer-Stiftung, and Tony Schönherr by Monbukagakusho is greatly acknowledged.

\section{REFERENCES}

1. Nawaz, A., M. Auweter-Kurtz, G. Herdrich, and H. L. Kurtz. 2006. Impulse measurements and thermal investigation of SIMP-LEX. AIAA Paper No. 2006-4855.

2. Bock, D., M. Auweter-Kurtz, H.L. Kurtz, and H.-P. Röser. 2006. Experimental investigation on thermal arcjet thruster development for a science mission to the Moon. 57th Astronautical Congress (International) Proceedings. IAC-06-C4403.

3. Laufer, R., M. Auweter-Kurtz, M. Lengowski, A. Nawaz, H.-P. Röeser, M. von Schoenermark, and H. Wagner. 2004. An all electrical small satellite for a technology demonstration and science mission to the Moon. 55th Astronautical Congress (International) of the International Astronautical Federation. IAC-04IAF-Q.2.b.05.

4. Palumbo, D. J. 1979. Solid propellant pulsed plasma propulsion system development for N-S stationkeeping. AIAA Paper No. 79-2097.

5. Zakrzwski, C., P. Sanneman, T. Hunt, and K. Blackman. 2001. Design of the EO-1 pulsed plasma thruster attitude control experiment. AIAA Paper No. 2001-3637.

6. Nawaz, A., R. Albertoni, G. Herdrich, and M. Auweter-Kurtz. 2008. Thrust efficiency optimimization of a pulsed plasma thruster. 26th Symposium (International) on Space Technology and Science. ISTS 2008-b-47.

7. Schönherr, T., A. Nawaz, G. Herdrich, H.-P. Röser, and M. Auweter-Kurtz. 2009. Influence of electrode shape on performance of pulsed magnetoplasmadynamic thruster SIMP-LEX. J. Propul. Power 25(2):380-86. 
8. Zube, D., I. Masuda, and Y. Gotoh. 1998. Low bus voltage hydrazine arcjet system for geostationary satellites. AIAA Paper No.1998-3631.

9. Bromaghim, D., J. R. LeDuc, R. M. Salasovich, G. G. Spanjers, J. M. Fife, M. J. Dulligan, J. H. Schilling, D. White, and L. K. Johnson. 2002. Electric propulsion space experiment (ESEX) on-orbit results. J. Spacecrafts Rockets 39(2):17718.

10. Bock, D., G. Herdrich, H.-P. Röser, H. Kurtz, and M. Auweter-Kurtz. 2007. An advanced ammonia propellant feed system for the thermal arcjet TALOS. AIAA Paper No. 2007-5186.

11. Brandner, J. J., E. Anurjew, L. Bohn, E. Hansjosten, T. Henning, U. Schygulla, A. Wenka, and K. Schubert. 2007. Concepts and realization of microstructure heat exchangers for enhanced heat transfer. J. Exper. Thermal Fluid Sci. 30(8):801-9. 